\title{
Expression of ubiquitin-conjugating enzyme E2T in colorectal cancers and clinical implications
}

\author{
XIANGTIAN WU ${ }^{1 *}$, GANG LIU ${ }^{2 *}$, RUITING LIU ${ }^{1}$, JING HE$^{3}$, GUORONG WANG ${ }^{1}$, \\ HAIBAO ZHANG ${ }^{4}$, TIANJIE LIU ${ }^{4}$, JIRONG BAI $^{5}$, NING CHENG $^{1}$ and JIAN QIU ${ }^{1}$ \\ ${ }^{1}$ Department of General Surgery, Shaanxi Provincial People's Hospital, Xi'an, Shaanxi 710061; \\ ${ }^{2}$ Department of Emergency Surgery, East Hospital, Tongji University, Shanghai 200120, P.R. China; \\ ${ }^{3}$ Department of Pathology, University of Texas Medical Branch, Galveston, TX 77555, USA; \\ ${ }^{4}$ Department of Urology, The First Affiliated Hospital of Xi'an Jiaotong University, Xi'an, Shaanxi 710000, \\ P.R. China; ${ }^{5}$ Department of Pathology, University of Nebraska Medical Center, Omaha, NE 68198, USA
}

Received April 19, 2020; Accepted August 13, 2020

DOI: $10.3892 / \mathrm{ol} .2020 .12138$

\begin{abstract}
Ubiquitin-conjugating enzyme E2T (UBE2T) plays a significant role in carcinogenesis. Previous studies have demonstrated that UBE2T promotes the development and progression of numerous types of cancer. However, the association between UBE2T expression and colorectal cancer (CRC) remains unclear. In the present study, UBE2T protein expression was examined in the tissues of patients with CRC via immunohistochemistry. In addition, UBE2T expression data and corresponding clinical information were obtained from The Cancer Genome Atlas (TCGA). In the clinical samples, the associations between UBE2T expression and clinicopathological factors were evaluated by the $\chi^{2}$ or Fisher's exact tests. In TCGA data, associations between UBE2T expression and clinical characteristics were evaluated using a logistic regression model. Overall survival was analyzed using Kaplan-Meier and Cox regression analyses. Reverse transcription-quantitative PCR (RT-qPCR) and western blotting assays were used to examine UBE2T expression in normal and CRC cell lines. Gene set enrichment analysis (GSEA) was performed on the dataset from TCGA. UBE2T protein was highly expressed in the cytoplasm of tumor cells in 29/50 clinical samples, whereas in the adjacent normal tissues, it was only highly expressed in 2/50 samples. Furthermore, UBE2T expression was associated with the $\mathrm{N}$ classification $(\mathrm{P}<0.001)$, clinical TNM stage $(\mathrm{P}<0.001)$ and histological grade of tumors $(\mathrm{P}=0.010)$. Survival analysis showed an association between
\end{abstract}

Correspondence to: Professor Guorong Wang, Department of General Surgery, Shaanxi Provincial People's Hospital, 256 West Youyi Road, Xi'an, Shaanxi 710061, P.R. China

E-mail: wangguorong66@126.com

${ }^{*}$ Contributed equally

Key words: ubiquitin-conjugating enzyme E2T, ubiquitination, colorectal cancer, prognosis high UBE2T expression and poor survival rate in patients with $\mathrm{CRC}(\mathrm{P}=0.002)$. Cox regression analysis also revealed that UBE2T expression was an independent prognostic factor for these patients $(\mathrm{P}=0.006)$. RT-qPCR and western blotting showed that UBE2T was expressed in CRC cell lines at higher levels than that in a normal colon cell line. Analysis of TCGA data revealed that UBE2T was highly expressed in tumor samples compared with normal samples, but was not associated with prognosis. GSEA showed that high expression of UBE2T was associated with the Kyoto Encyclopedia of Genes and Genomes pathways 'cell cycle', 'oxidative phosphorylation', 'DNA replication', 'p53 signaling pathway', ‘ubiquitin mediated proteolysis' and 'pentose phosphate pathway'. These results indicate that UBE2T may play an important role in the progression of CRC and serve as a potential prognostic factor during the treatment of cancer.

\section{Introduction}

Colorectal cancer (CRC) is one of the most commonly diagnosed malignant tumors worldwide $(1,2)$. The latest statistics indicate that $\mathrm{CRC}$ has the third highest incidence among all malignant tumors (3). Moreover, CRC has the second highest fatality rate among all malignant tumors, and the morbidity and mortality rates of CRC are continuing to rise (2). Due to the lack of effective treatments for CRC, more research efforts need to be focused on determining the molecular mechanisms of its oncogenesis. With such research, improved antitumor agents and immunotherapies aimed at novel specific molecular targets could be developed (4).

Ubiquitin-conjugating enzyme E2T (UBE2T) is a member of the ubiquitin-conjugating (E2) enzyme family that plays an important role in the ubiquitin-proteasome pathway. The proteasome pathway participates in a number of crucial cell activities, such as the repair of DNA damage, differentiation, apoptosis, angiogenesis, inflammation and various cancer-associated processes (5). Together with E3 ubiquitin ligase, the E2 enzyme family has been reported to serve roles in various types of cancer, such as breast cancer (6). As a component of the Fanconi anemia (FA) pathway, UBE2T 
participates in DNA damage and repair pathways. These pathways are recognized to be crucial in the tumorigenesis of lung, breast, prostate, gastric and nasopharyngeal cancer (7-9). Furthermore, it has been revealed that UBE2T gene silencing can lead to FA and that this silencing can increase the sensitivity of tumor cells to cross-linking agents used to target the DNA damage-repair response (7). Therefore, UBE2T may have an important role in the maintenance of genome integrity. However, the role of UBE2T in CRC remains to be elucidated. Therefore, the aim of the present study was to examine UBE2T expression in patients with CRC and evaluate its prognostic significance by identifying associations between its expression and various clinicopathological parameters of $\mathrm{CRC}$, and to verify the role of UBE2T in CRC using The Cancer Genome Atlas (TCGA) database.

\section{Materials and methods}

Human tissue samples and cell lines. For the purpose of this study, a total of 50 paired surgically resected specimens of fresh colorectal adenocarcinoma and adjacent normal tissues ( $>5 \mathrm{~cm}$ away from the tumor edge) were collected from patients between April 2012 and April 2013 in Shaanxi Provincial People's Hospital (Shaanxi, China). After surgical resection, the tissues were immediately frozen in liquid nitrogen and then transferred to a $-80^{\circ} \mathrm{C}$ refrigerator. Samples were collected from 29 male and 21 female patients, and the diagnoses of all patients were confirmed by pathological examination. Only patients who had not received any radiotherapy or chemotherapy prior to surgery and who had a complete clinical data record were included in this study. All patients were followed up for $\geq 5$ years by telephone. All specimens were classified according to the 8th edition Tumor Node Metastasis (TNM) staging system enacted by The International Union Against Cancer and American Joint Committee on Cancer (10). The human normal colon mucosal cell line NCM460 and human colorectal adenocarcinoma cell lines SW480, SW620, Caco2, HCT116, HT-29, LoVo and RKO were purchased from the American Type Culture Collection and maintained in RPMI-1640 medium (Gibco; Thermo Fisher Scientific, Inc.) containing $10 \%$ fetal bovine serum (Gibco; Thermo Fisher Scientific, Inc.) and $1 \%$ penicillin/streptomycin (Thermo Fisher Scientific) at $37^{\circ} \mathrm{C}$ under $5 \% \mathrm{CO}_{2}$ in Xi'an Jiaotong University First Affiliated Hospital Urology Research Center Laboratory.

Bioinformatics analysis. Gene expression data and corresponding clinical data were obtained from TCGA database (https://portal.gdc.cancer.gov). The parameters were set as follows: Primary Site: Colon, rectum; Program: TCGA; Project: TCGA-COAD, TCGA-READ; Data Category: Transcriptome profiling; Workflow Type: HTSeq-FPKM. In total, 612 samples (568 tumor samples and 44 normal samples) were obtained by this search, and all the samples were downloaded in June 2020. The gene expression data of UBE2T were extracted to perform both non-paired and paired differential expression analyses between tumor and normal tissues. Patients were divided into the UBE2T-high and UBE2T-low groups based on the median of UBE2T expression level. Logistic regression analysis, as well as univariate and multivariate
Cox regression analyses were performed to investigate the associations between UBE2T expression and the clinical characteristics of the patients. Survival analysis was conducted to explore whether UBE2T expression influences the prognosis of patients with CRC. Gene Set Enrichment Analysis (GSEA) was performed to identify the Kyoto Encyclopedia of Genes and Genomes (KEGG) pathways according to their association with UBE2T expression using GSEA software v3.0 (Broad Institute). The C2 (c2.cp.kegg.v7.1.symbols.gmt) sub-collection was chosen as the reference gene set and gene set permutations were performed 1,000 times for the analysis. A false-discovery rate (FDR) q value $<0.05$ was considered to indicate a statistically significant result.

Immunohistochemistry (IHC). For IHC analysis, fresh CRC and normal adjacent tissue were fixed in $4 \%$ paraformaldehyde solution at room temperature for $48 \mathrm{~h}$. Next, tissues were embedded in paraffin, and 4- $\mu \mathrm{m}$ tissue slices were mounted on slides, baked, dewaxed with xylene and hydrated by a gradient ethanol series with 5-min washes at room temperature as follows: 100, 100, 95, 95, 80, 70 and 50\% ethanol, followed by two washes with $\mathrm{ddH}_{2} \mathrm{O}$. The slides were then placed in citric acid buffer for antigen retrieval, heated under high-pressure at $120^{\circ} \mathrm{C}$ for $5 \mathrm{~min}$ and then allowed to cool to room temperature. Endogenous peroxidase activity was blocked by immersing the samples in $3 \% \mathrm{H}_{2} \mathrm{O}_{2}$-methanol for $30 \mathrm{~min}$ at room temperature. To eliminate non-specific staining, slides were incubated with 5\% bovine serum albumin (Beijing Solarbio Science \& Technology Co., Ltd.) at room temperature for $30 \mathrm{~min}$. Next, slides were incubated with the primary rabbit polyclonal anti-UBE2T antibody (cat. no. 10105-2-AP; 1:1,000; ProteinTech Group, Inc.) at $4^{\circ} \mathrm{C}$ overnight. The next day, slides were incubated with the secondary biotinylated antibody (cat. no. GK600510; original liquid; Gene Tech Co., Ltd.) for $1 \mathrm{~h}$ at room temperature. Finally, slides were stained with 3,3'-diaminobenzidine tetrahydrochloride at room temperature for $25 \mathrm{sec}$ and counterstained with hematoxylin at room temperature for $5 \mathrm{~min}$. The negative control was prepared by omitting the primary antibody. The slides were observed by light microscopy under x200 magnification.

Semi-quantitative analysis of IHC results. IHC staining was evaluated independently by two senior pathologists, who were blinded to the clinical data. The slides with disagreements were evaluated by a third pathologist, and the final results were subject to two matching opinions. The immunoreactive score was calculated based on the percentage of stained cells as well as the staining intensity. The intensity of staining was classified as follows: i) 0 , no staining; ii) 1 , weak staining; iii) 2 , medium staining; iv) 3 , strong staining. The area of staining was graded as $0(0 \%), 1(1-25 \%), 2(26-50 \%), 3(51-75 \%)$ or $4(76-100 \%)$. Finally, patients were divided into two groups: Low UBE2T expression group ( $\leq 3)$ and high UBE2T expression group (>3) according to the sum of the intensity and area scores.

Reverse transcription-quantitative PCR (RT-qPCR). To determine mRNA expression levels in the cell lines, total RNA from the cell cultures was isolated using TRIzol ${ }^{\circledR}$ reagent (Thermo Fisher Scientific, Inc.), reverse transcribed to cDNA using the PrimeScript $^{\mathrm{TM}}$ RT reagent kit (Takara Biotechnology Co., 

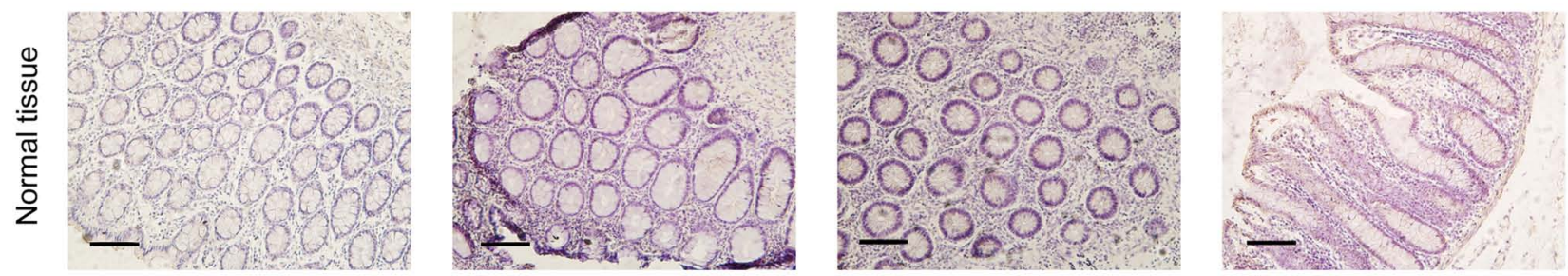

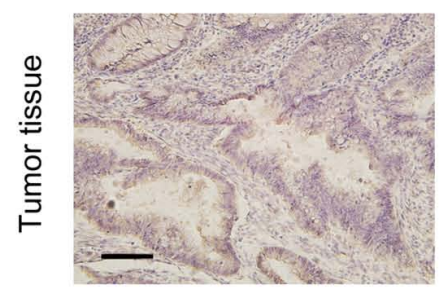

$\mathrm{T} 1$



$\mathrm{T} 2$



T3



$\mathrm{T} 4$

Figure 1. UBE2T protein expression in colorectal cancer and adjacent normal tissue samples examined by immunohistochemistry. UBE2T expression at different invasion depths is shown. Scale bar, $250 \mu \mathrm{m}$ (magnification, x200). UBE2T, ubiquitin-conjugating enzyme E2T; T1-T4, tumor invasion depth.

Ltd.) for $15 \mathrm{~min}$ at $60^{\circ} \mathrm{C}$ and $5 \mathrm{sec}$ at $85^{\circ} \mathrm{C}$, and stored at $4^{\circ} \mathrm{C}$ qPCR was performed on a CFX96 Real-Time PCR Detection system (Bio-Rad Laboratories, Inc.) with SYBR-Green PCR Master Mix (Takara Biotechnology Co., Ltd.). The primer sequences used were as follows: UBE2T forward 5'-AGCCAC CCCCAGGCATCACA-3' and reverse, 5'-TCTCCAAGCACC TTTTGGTGGCA-3'; and GAPDH forward, 5'-ACCCAGAAG ACTGTGGATGG-3' and reverse, 5'-TGCTGTAGCCAAATT CGTTG-3'. The thermocycling conditions were as follows: $95^{\circ} \mathrm{C}$ for $30 \mathrm{sec}$, followed by 40 cycles of $95^{\circ} \mathrm{C}$ for $5 \mathrm{sec}$ and $60^{\circ} \mathrm{C}$ for $30 \mathrm{sec}$, and dissociation at $60^{\circ} \mathrm{C}$ for $1 \mathrm{~min}$ and $95^{\circ} \mathrm{C}$ for $1 \mathrm{sec}$. UBE2T mRNA expression was quantified using the $2^{-\triangle \Delta C q}$ method and normalized to the internal reference gene GAPDH (11).

Western blot analysis. For western blotting, cultured cells were washed with PBS, and total protein was extracted using radioimmunoprecipitation lysis buffer (Shaanxi Zhonghui Hecai Biomedical Technology Co., Ltd.) and protease inhibitors. A bicinchoninic acid assay kit (Beyotime Institute of Biotechnology) was used to determine the protein concentration. Next, protein samples (30 $\mu \mathrm{g} / \mathrm{lane})$ were separated via SDS-PAGE on a $12 \%$ gel, and subsequently transferred to a polyvinylidene fluoride membrane (Immobilon; EMD Millipore). After blocking with 5\% non-fat milk at room temperature for $2 \mathrm{~h}$, membranes were incubated at $4^{\circ} \mathrm{C}$ overnight with primary rabbit polyclonal anti-UBE2T (cat. no. 10105-2-A; 1:1,000; ProteinTech Group, Inc.) and anti-GAPDH (cat. no. KC-5G4; 1:10,000; Kang Chen Biotech, Inc.) antibodies. Membranes were then incubated with horseradish peroxidase-conjugated AffiniPure goat anti-rabbit IgG (cat. no. ZB-2301; 1:2,000; OriGene Technologies, Inc.) for $1 \mathrm{~h}$ at room temperature. Finally, membranes were washed in Tris-buffered saline with Tween-20 and protein bands were detected using the Molecular Imager ChemiDoc XRS system (Bio-Rad Laboratories, Inc.). The intensity of the UBE2T protein bands was determined using ImageJ software (v1.52a; National Institutes of Health), and UBE2T expression was normalized to GAPDH.
Statistical analysis. SPSS 22.0 (IBM Corp.) software and GraphPad Prism 8.0.2 (GraphPad Software, Inc.) were used for statistical analysis. A two-sided $\mathrm{P}<0.05$ was considered to indicate statistical significance. The $\chi^{2}$ test or Fisher's exact test was used to analyze the associations between clinicopathological characteristics and UBE2T expression. The Kaplan-Meier method and log-rank test were used to perform survival analyses. The effects of clinicopathological variables and UBE2T expression on survival were analyzed by univariate and multivariate analyses using a Cox proportional hazard regression model. Clinical variables that were found to be statistically significant $(\mathrm{P}<0.05)$ in the univariate analysis were incorporated into the multivariate analysis. One-way analysis of variance was used to analyze differences in relative UBE2T mRNA and protein expression levels among the cell lines (both $\mathrm{P}<0.05$ ), followed by Dunnett's multiple comparisons test. Mann-Whitney U test and Wilcoxon's matched pairs tests were used to perform non-paired and paired differential expression analyses, respectively, for tumor and normal tissues.

\section{Results}

UBE2T expression and its associations with clinicopathological characteristics in patients with CRC. The 50 patients included in the study ranged from 30 to 90 years in age, and had a median age at diagnosis of 67 years. To evaluate UBE2T protein expression in CRC tissues, paraffin-embedded samples from the 50 patients with CRC were examined by IHC. The results revealed that UBE2T protein was highly expressed in the cytoplasm of the tumor cells in 29/50 samples and in $2 / 50$ adjacent normal samples (Fig. 1). The $\chi^{2}$ analysis of the IHC scoring results revealed that UBE2T expression in CRC tissue was significantly higher compared with that in adjacent normal tissue $(\mathrm{P}<0.001)$. Moreover, the $\chi^{2}$ or Fisher's exact tests were performed to evaluate the associations between low and high UBE2T expression in CRC tissue and clinicopathological parameters of CRC. Based on this analysis, UBE2T expression was found to be positively associated with the $\mathrm{N}$ classification $(\mathrm{P}<0.001)$, clinical TNM stage $(\mathrm{P}<0.001)$ and 


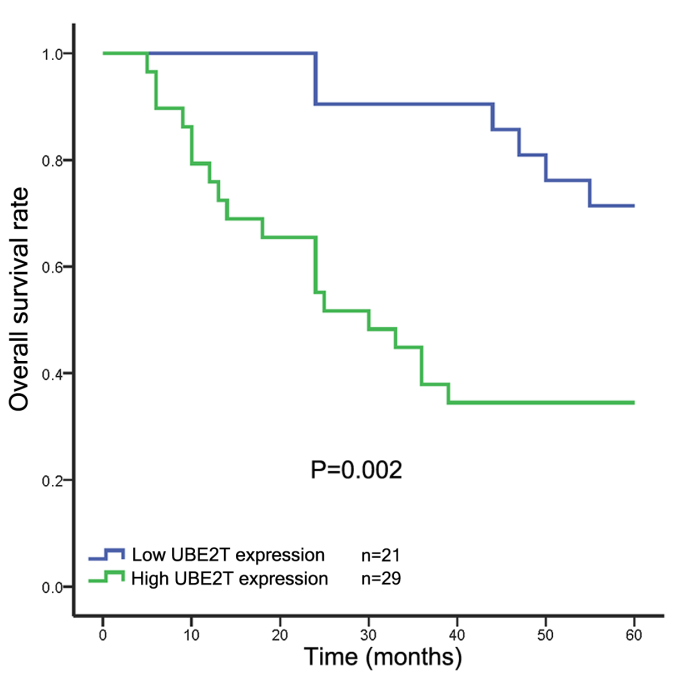

Figure 2. Kaplan-Meier survival curves for 50 patients with colorectal cancer according to UBE2T protein expression in tumor tissue. There was a significant difference in survival between patients with low and high expression of UBE2T. UBE2T, ubiquitin-conjugating enzyme E2.

histological grade $(\mathrm{P}=0.010)$. However, no association was observed between UBET2 protein expression and sex, age, tumor size, T classification or M classification (Table I).

Survival analysis of clinical patients. The results of Kaplan-Meier analysis and a log-rank test indicated that patients with high levels of UBE2T protein expression in their tumors had a significantly shorter overall survival time compared with those with low UBE2T expression (Fig. 2). Additionally, univariate and multivariate Cox regression analyses were performed to explore the independent prognostic factors for the overall survival of patients with CRC. Univariate Cox regression analyses indicated that UBE2T expression $(\mathrm{P}=0.006), \mathrm{N}$ classification $(\mathrm{P}=0.043), \mathrm{M}$ classification $(\mathrm{P}=0.012)$ and clinical $\mathrm{TNM}$ stage $(\mathrm{P}=0.008)$ were associated with the overall survival of patients with CRC. Additionally, multivariate Cox regression analysis suggested that UBE2T expression $(\mathrm{P}=0.018)$ and $\mathrm{M}$ classification $(\mathrm{P}=0.040)$ were both independent predictors of overall survival, indicating that UBE2T protein expression could be useful in the prediction of overall survival for patients with CRC (Table II).

UBE2T $m R N A$ and protein expression levels are elevated in $C R C$ cell lines. To examine the expression levels of UBE2T mRNA and protein in normal colon and CRC cell lines, RT-qPCR and western blotting were performed. The expression of UBE2T was examined in the CRC cell lines SW480, SW620, Caco2, HCT116, HT-29, LoVo and RKO, as well as in the normal colonic mucosa cell line NCM460. UBE2T mRNA and protein expression levels varied among the different cell lines. In the CRC cell lines, UBE2T expression was observed to increase in the following order: Caco2, RKO, HCT116, SW480, LoVo, HT-29 and SW620. Significantly lower UBE2T expression was detected at both the mRNA and protein levels in the normal colonic mucosa cell line NCM460 compared with the CRC cell lines (Fig. 3). Therefore, based on the IHC of patient samples and the RT-qPCR and western blot analysis
Table I. Associations between UBE2T protein expression and clinical parameters in patients with CRC.

\begin{tabular}{|c|c|c|c|c|}
\hline \multirow[b]{2}{*}{ Clinical parameters } & \multirow[b]{2}{*}{$\mathrm{N}$} & \multicolumn{2}{|c|}{ UBE2T expression } & \multirow[b]{2}{*}{ P-value } \\
\hline & & Low & High & \\
\hline Sex & & & & 0.206 \\
\hline Male & 29 & 10 & 19 & \\
\hline Female & 21 & 11 & 10 & \\
\hline Age, years & & & & 0.196 \\
\hline$>60$ & 33 & 16 & 17 & \\
\hline$\leq 60$ & 17 & 5 & 12 & \\
\hline Tumor size, $\mathrm{cm}$ & & & & 0.179 \\
\hline$>4$ & 23 & 12 & 11 & \\
\hline$\leq 4$ & 27 & 9 & 18 & \\
\hline Histological grade & & & & $0.010^{\mathrm{a}}$ \\
\hline High & 5 & 5 & 0 & \\
\hline Moderate/poor & 45 & 16 & 29 & \\
\hline T classification & & & & 0.068 \\
\hline $\mathrm{T} 1+\mathrm{T} 2$ & 3 & 3 & 0 & \\
\hline $\mathrm{T} 3+\mathrm{T} 4$ & 47 & 18 & 29 & \\
\hline $\mathrm{N}$ classification & & & & $<0.001^{\mathrm{a}}$ \\
\hline No & 19 & 15 & 4 & \\
\hline $\mathrm{N} 1+\mathrm{N} 2$ & 31 & 6 & 25 & \\
\hline M classification & & & & 0.383 \\
\hline M0 & 45 & 20 & 25 & \\
\hline M1 & 5 & 1 & 4 & \\
\hline Clinical TNM stage & & & & $<0.001^{\mathrm{a}}$ \\
\hline $\mathrm{I}+\mathrm{II}$ & 18 & 14 & 4 & \\
\hline III+IV & 32 & 7 & 25 & \\
\hline
\end{tabular}

${ }^{\mathrm{a}} \mathrm{P}<0.05$.UBE2T, ubiquitin-conjugating enzyme E2T; $\mathrm{CRC}$, colorectal cancer; $\mathrm{T}$, tumor; $\mathrm{N}$, node; $\mathrm{M}$, metastasis.

of cell lines, it can be concluded that UBE2T is primarily expressed in CRC tissues and cell lines rather than in normal colorectal tissues and cells. Based on the UBE2T expression detected in the CRC cell lines in the present study, SW620 cells were selected for further experiments, as they exhibited the highest expression of UBE2T.

UBE2T expression and its clinical significance in CRC in $T C G A$. The non-paired differential expression analysis of the 568 tumor tissues and 44 normal tissues from TCGA indicated that there was a significant difference in the level of UBE2T expression between tumor and normal tissues $(\mathrm{P}<0.001$; Fig. 4A). As shown in Fig. 4B, a comparable result was obtained from the paired differential expression analysis of the tumor and normal tissues from 44 patients. After removing incomplete clinical data, the clinical data of 480 patients with $\mathrm{CRC}$ and the corresponding UBE2T expression were subjected to logistic regression analyses to investigate the potential associations between them. Among the 480 patients, there were 226 females and 254 males, and the median age at diagnosis was 68 years. As shown in Table III, UBE2T expression as 
Table II. Survival analysis of patients with CRC using univariate and multivariate Cox regression analyses.

\begin{tabular}{|c|c|c|c|c|c|c|}
\hline \multirow[b]{2}{*}{ Clinical parameters } & \multicolumn{3}{|c|}{ Univariate analysis } & \multicolumn{3}{|c|}{ Multivariate analysis } \\
\hline & P-value & HR & $95 \% \mathrm{CI}$ & P-value & HR & $95 \% \mathrm{CI}$ \\
\hline $\begin{array}{l}\text { Age, years } \\
\leq 60 \text { vs. }>60\end{array}$ & 0.837 & 0.916 & $\begin{array}{l}0.395 \\
2.122\end{array}$ & & & \\
\hline $\begin{array}{l}\text { Sex } \\
\text { Male vs. female }\end{array}$ & 0.786 & 1.117 & $\begin{array}{l}0.502 \\
2.488\end{array}$ & & & \\
\hline $\begin{array}{l}\text { Tumor size, } \mathrm{cm} \\
\leq 4 \text { vs. }>4\end{array}$ & 0.099 & 0.501 & $\begin{array}{l}0.221 \\
1.138\end{array}$ & & & \\
\hline $\begin{array}{l}\text { Histological grade } \\
\text { Poor/moderate vs. high }\end{array}$ & 0.156 & 1.713 & $\begin{array}{l}0.815 \\
3.603\end{array}$ & & & \\
\hline $\begin{array}{l}\text { T classification } \\
\text { T3+T4 vs. T1+T2 }\end{array}$ & 0.163 & 1.607 & $\begin{array}{l}0.826 \\
3.126\end{array}$ & & & \\
\hline $\begin{array}{l}\text { Clinical TNM stage } \\
\text { III+IV vs. I+II }\end{array}$ & $0.008^{\mathrm{a}}$ & 2.310 & $\begin{array}{l}1.238 \\
4.309\end{array}$ & 0.604 & 0.735 & $\begin{array}{l}0.229 \\
2.352\end{array}$ \\
\hline $\begin{array}{l}\mathrm{N} \text { classification } \\
\mathrm{N} 1+\mathrm{N} 2 \text { vs. N0 }\end{array}$ & $0.043^{\mathrm{a}}$ & 1.696 & $\begin{array}{l}1.017 \\
2.827\end{array}$ & 0.297 & 1.525 & $\begin{array}{l}0.691 \\
3.366\end{array}$ \\
\hline $\begin{array}{l}\text { M classification } \\
\text { M1 vs. M0 }\end{array}$ & $0.012^{\mathrm{a}}$ & 4.098 & $\begin{array}{r}1.370 \\
12.260\end{array}$ & $0.040^{\mathrm{a}}$ & 8.109 & $\begin{array}{r}1.103 \\
59.628\end{array}$ \\
\hline $\begin{array}{l}\text { UBE2T expression } \\
\text { High vs. low }\end{array}$ & $0.006^{\mathrm{a}}$ & 3.676 & $\begin{array}{l}1.458 \\
9.269\end{array}$ & $0.018^{\mathrm{a}}$ & 3.683 & $\begin{array}{r}1.255 \\
10.813\end{array}$ \\
\hline
\end{tabular}

Multivariate analysis included only those variables that were significantly associated with survival $(\mathrm{P}<0.05)$. ${ }^{\mathrm{a}}<<0.05$. UBE2T, ubiquitinconjugating enzyme E2T; CRC, colorectal cancer; T, tumor; N, node; M, metastasis; HR, hazard ratio; CI, confidence interval.
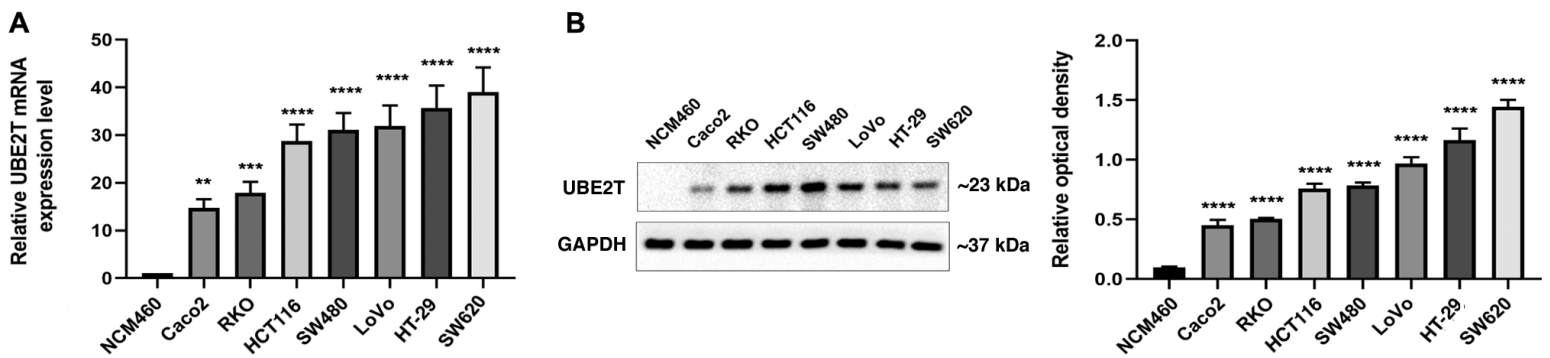

Figure 3. UBE2T expression in CRC and normal colorectal cell lines. (A) Reverse transcription-quantitative PCR analysis of UBE2T mRNA expression and (B) western blotting and densitometric analysis of UBE2T protein expression in various human CRC cell lines and one normal colorectal cell line. GAPDH was used as an internal control. Data are presented as the mean $\pm \mathrm{SD}(\mathrm{n}=3){ }^{* * *} \mathrm{P}<0.01,{ }^{* * * *} \mathrm{P}<0.001$ and ${ }^{* * * * *} \mathrm{P}<0.0001$ vs. NCM460. UBE2T, ubiquitin-conjugating enzyme E2T; CRC, colorectal cancer.
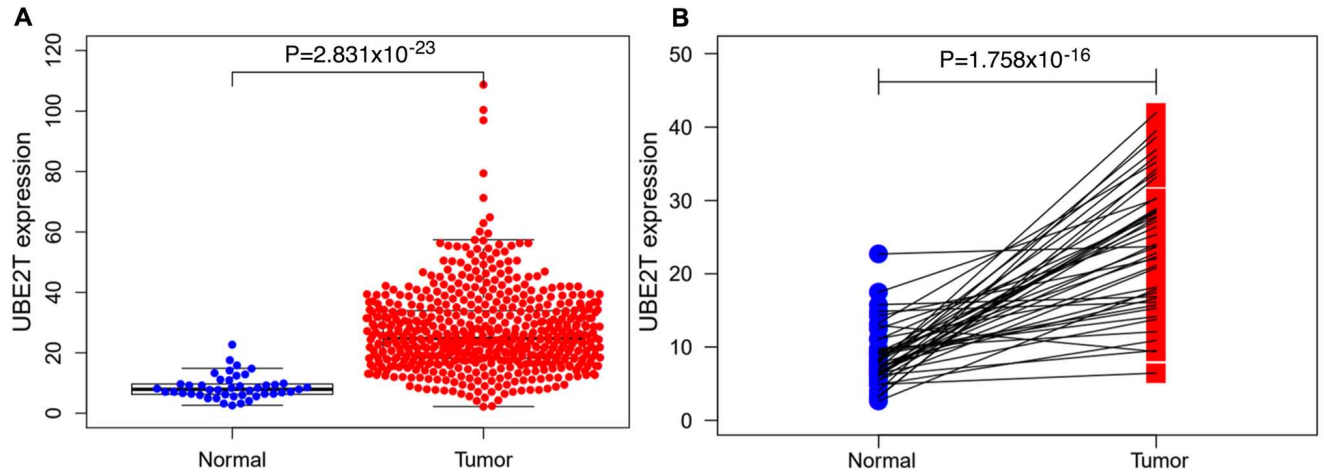

Figure 4. UBE2T expression in colorectal cancer based on TCGA data. (A) UBE2T expression in 568 tumor and 44 normal tissues from TCGA database. (B) UBE2T expression in 44 paired tumor and normal tissues from TCGA database. UBE2T, ubiquitin-conjugating enzyme E2T; TCGA, The Cancer Genome Atlas. 
Table III. Logistic regression analysis of the association between UBE2T expression and clinicopathological characteristics in patients with CRC from TCGA.

\begin{tabular}{lccc}
$\begin{array}{l}\text { Clinicopathological } \\
\text { characteristics }\end{array}$ & OR & $95 \%$ CI & P-value \\
\hline Stage & & & \\
$\quad$ II vs. I & 1.024 & $1.003-1.046$ & $0.029^{\mathrm{a}}$ \\
III vs. I & 1.008 & $0.984-1.031$ & 0.528 \\
$\quad$ IV vs. I & 1.005 & $0.980-1.032$ & 0.682 \\
T classification & & & \\
T2 vs. T1 & 0.960 & $0.926-0.996$ & $0.030^{\mathrm{a}}$ \\
T3 vs. T1 & 0.979 & $0.949-1.010$ & 0.188 \\
T4 vs. T1 & 0.968 & $0.932-1.005$ & 0.093 \\
N classification & & & \\
N1 vs. N0 & 0.981 & $0.964-0.999$ & $0.043^{\mathrm{a}}$ \\
N2 vs. N0 & 0.996 & $0.977-1.015$ & 0.667 \\
M classification & & & \\
M1 vs. M0 & 0.991 & $0.972-1.011$ & 0.373 \\
\hline
\end{tabular}

${ }^{\mathrm{a}} \mathrm{P}<0.05$. UBE2T, ubiquitin-conjugating enzyme E2T; $\mathrm{CRC}$, colorectal cancer; TCGA, The Cancer Genome Atlas; T, tumor; N, node; $\mathrm{M}$, metastasis; OR, odds ratio; CI, confidence interval.

a categorical dependent variable (based on a median expression of 25.01) was significantly associated with stage [II vs. I; odds ratio $(\mathrm{OR})=1.024, \mathrm{P}=0.029]$, $\mathrm{T}$ classification $(\mathrm{T} 2$ vs. $\mathrm{T} 1$; $\mathrm{OR}=0.960, \mathrm{P}=0.030)$ and lymph node metastasis (N1 vs. N0; $\mathrm{OR}=0.981, \mathrm{P}=0.043$ ).

Survival analysis and Cox regression analysis in TCGA. Kaplan-Meier survival analysis showed that there was no significant difference in prognosis between patients in the UBE2T-high and UBE2T-low groups $(\mathrm{P}=0.238$; data not shown). Furthermore, the same conclusion could be drawn from the results of the univariate and multivariate Cox regression analyses (Table IV). Excluding the influence of other confounding factors, the expression level of UBE2T was still not associated with the prognosis patients with CRC; however, age and $\mathrm{T}$ stage were associated with prognosis.

Identification of UBE2T-associated pathways via GSEA. GSEA was conducted on the UBE2T-high and UBE2T-low groups to explore the underlying mechanisms of UBE2T in CRC. As shown in Fig. 5, high expression of UBE2T was associated with the KEGG pathways 'cell cycle', 'oxidative phosphorylation', 'DNA replication', 'p53 signaling pathway', 'ubiquitin mediated proteolysis' and 'pentose phosphate pathway'.

\section{Discussion}

$\mathrm{CRC}$ is one of the most common cancers, and although great progress has been achieved in terms of its diagnosis and treatment, the rate of overall survival for patients with advanced CRC remains low (12). Several tumor markers are used for the diagnosis of CRC and function as targets for antitumor

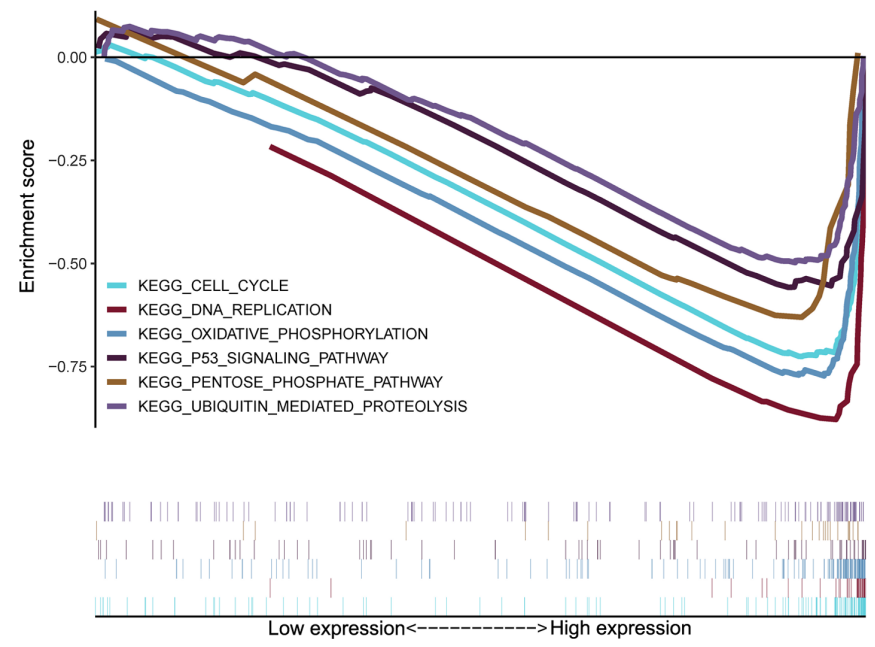

Figure 5. Gene set enrichment analysis of ubiquitin-conjugating enzyme E2T expression. KEGG, Kyoto Encyclopedia of Genes and Genomes.

therapy, including adenomatous polyposis coli protein (APC), carcinoembryonic antigen, carbohydrate antigen 199, vascular endothelial growth factor and p53; however, their sensitivity and specificity for the diagnosis and prognosis of CRC are not high (13-15). Hence, the molecular mechanisms associated with the development and progression of CRC must be further explored in order to find potentially useful markers for early CRC diagnosis and disease monitoring (16).

The ubiquitin-proteasome system, which consists of two E1 ubiquitin-activating enzymes, 40 E2 enzymes and $>600$ E3 ubiquitin ligases, plays a crucial role in numerous cellular activities, most importantly in the degradation of intracellular proteins $(17,18)$. As a member of the E2 enzyme family, UBE2T has gained considerable attention for its roles in the promotion of tumorigenesis and mediation of several signaling pathways (19). For example, reported that ubiquitin E2 has been reported to stabilize $\beta$-catenin, one of the key regulators of the Wnt/ $\beta$-catenin signaling pathway, by mediating $\beta$-catenin polyubiquitination to form ubiquitin- $\beta$-catenin conjugates (6). In addition, Rickman et al (7) demonstrated that UBE2T is a crucial member of the FA pathway (20), and Longerich et al (21) reported that it mediates the mono-ubiquitination of Fanconi anemia complementation group L and Fanconi anemia complementation group I. In addition to its role in the FA pathway, UBE2T has other important functions in cells (22). For instance, the interaction of UBE2T with the breast cancer type 1 susceptibility protein (BRCA1)/BRCA1 associated RING domain 1 complex and high expression levels of UBE2T has been demonstrated to be critical in the progression of breast cancer (23). According to other studies, UBE2T expression is also upregulated in other types of tumor, including gastric, lung, prostate, nasopharyngeal and bladder cancer (24-27). However, few studies have examined the association between UBE2T and CRC; one study has reported that UBE2T may promote CRC progression (28). Based on previous reports, we speculated that the role of UBE2T may be similar in different types of tumor and that UBE2T could be involved in the development and progression of CRC. 
Table IV. Univariate and multivariate analyses of the associations between UBE2T expression and overall survival in patients with CRC from TCGA.

\begin{tabular}{|c|c|c|c|c|c|c|}
\hline \multirow[b]{2}{*}{ Parameters } & \multicolumn{3}{|c|}{ Univariate analysis } & \multicolumn{3}{|c|}{ Multivariate analysis } \\
\hline & HR & $95 \% \mathrm{CI}$ & P-value & HR & $95 \% \mathrm{CI}$ & $\mathrm{P}$-value \\
\hline Age (continuous) & 1.036 & $1.014-1.057$ & $0.001^{\mathrm{a}}$ & 1.046 & $1.024-1.069$ & $0.001^{\mathrm{a}}$ \\
\hline Sex (male vs. female) & 1.025 & $0.668-1.573$ & 0.911 & 0.875 & $0.566-1.352$ & 0.546 \\
\hline Stage (IV vs. I) & 21.291 & $5.117-88.581$ & $0.001^{\mathrm{a}}$ & 1.527 & $0.753-3.095$ & 0.240 \\
\hline T classification (T4 vs. T1) & 8.895 & $1.191-66.464$ & $0.033^{\mathrm{a}}$ & 1.811 & $1.114-2.943$ & $0.017^{\mathrm{a}}$ \\
\hline N classification (N2 vs. N1) & 4.210 & $2.552-6.944$ & $0.001^{\mathrm{a}}$ & 1.208 & $0.785-1.857$ & 0.390 \\
\hline M classification (M1 vs. M0) & 4.698 & $3.029-7.285$ & $0.001^{\mathrm{a}}$ & 1.775 & $0.676-4.831$ & 0.238 \\
\hline UBE2T (continuous) & 0.998 & $0.981-1.014$ & 0.786 & 1.001 & $0.984-1.017$ & 0.952 \\
\hline
\end{tabular}

${ }^{\mathrm{a}} \mathrm{P}<0.05$. UBE2T, ubiquitin-conjugating enzyme E2T; CRC, colorectal cancer; TCGA, The Cancer Genome Atlas; T, tumor; N, node; M, metastasis; HR, hazard ratio; CI, confidence interval.

In the present study, the expression of UBE2T in CRC tissues and matched adjacent normal tissue samples was examined via IHC. The results suggested that UBE2T protein expression was higher in CRC tissues than in the surrounding normal tissue. Additionally, high expression of UBE2T protein was found to be significantly associated with high $\mathrm{N}$ classification, advanced clinical TNM stage and tumors of poor histological grade. Data from $>5$ years of follow-up of the patients included in the present study showed that patients with high UBE2T expression were more likely to have a worse overall survival outcome. Moreover, Cox proportional hazard regression analysis indicated that $\mathrm{M}$ classification and UBE2T expression were independent prognostic factors for CRC. In addition to UBE2T protein expression in patient tissue, UBE2T expression in various CRC cell lines and a normal colorectal cell line was also examined at the mRNA and protein levels. This in vitro analysis revealed that UBE2T was upregulated in CRC cells at the mRNA and protein levels compared with its expression in normal colorectal cells. TCGA dataset analysis revealed that UBE2T was highly expressed in tumors compared with normal samples, which was consistent with the results from patient tissues. However, UBE2T was not associated with CRC prognosis in TCGA data. GSEA analysis revealed that high expression of UBE2T was associated with 'cell cycle' and 'DNA replication', the abnormalities in which have been reported to be involved in oncogenesis $(29,30)$. Oxidative phosphorylation may be involved in cancer progression (31), which is consistent with the GSEA results of the present study. In addition, the p53 signaling pathway is involved in the development of CRC and can alter the risk of CRC (32). Additionally, aberrant ubiquitin-mediated proteolysis of proteins, especially cell cycle regulatory proteins, may promote tumorigenesis (33). During tumorigenesis, the role of the elevated pentose phosphate pathway is protection from cell death (34), which also supports the GSEA results obtained in the present study.

In summary, the present study demonstrated the upregulation of UBE2T expression in CRC and its association with patient prognosis, which is consistent with previous studies, thus suggesting that UBE2T may have a similar role in various types of cancer, such as breast cancer, hepatoma and cervical carcinoma (35). Based on the clinical results, it may be concluded that UBE2T is a valuable prognostic marker for $\mathrm{CRC}$ and could provide a novel direction for the development of targeted therapeutic strategies. However, there are limitations of this study, which may be addressed by increasing the clinical sample size, and examining the role of UBE2T in $\mathrm{CRC}$ tumorigenesis and the underlying associations between UBE2T and downstream molecules in further studies.

\section{Acknowledgements}

Not applicable.

\section{Funding}

This study was supported by the grants from the Innovation Capability Support Plan of Shaanxi Science and Technology Department-Science and Technology Innovation Team (grant no. 2020TD-048) and 2018 Shaanxi Province Health research fund program (grant no. 2018A003).

\section{Availability of data and materials}

The datasets used and/or analyzed during the current study are available from the corresponding author on reasonable request.

\section{Authors' contributions}

GW conceived the experiments. XW and GL designed the experiments. JQ prepared the samples. NC, JQ and RL performed the experiments. $\mathrm{JB}$ and $\mathrm{JH}$ processed and analyzed the data. $\mathrm{XW}$ and $\mathrm{JH}$ wrote the manuscript with input from all authors. $\mathrm{HZ}$ and TL performed the bioinformatics analysis and article revision. All authors read and approved the final manuscript.

\section{Ethics approval and consent to participate}

Informed consent was obtained from all patients involved in this study, which was conducted according to the guidelines 
and with the approval of the Medical Ethics Committee of Shaanxi Provincial People's Hospital.

\section{Patient consent for publication}

Not applicable.

\section{Competing interests}

The authors declare that they have no competing interests.

\section{References}

1. Siegel RL, Miller KD, Goding SA, Fedewa SA, Butterly LF, Anderson JC, Cercek A, Smith RA and Jemal A: Colorectal cancer statistics, 2020. CA Cancer J Clin 70: 145-164, 2020.

2. Siegel RL, Miller KD and Jemal A: Cancer statistics, 2019. CA Cancer J Clin 69: 7-34, 2019.

3. Bray F, Ferlay J, Soerjomataram I, Siegel RL, Torre LA and Jemal A: Global cancer statistics 2018: GLOBOCAN estimates of incidence and mortality worldwide for 36 cancers in 185 countries. CA Cancer J Clin 68: 394-424, 2018.

4. Custodio A and Feliu J: Prognostic and predictive biomarkers for epidermal growth factor receptor-targeted therapy in colorectal cancer: Beyond KRAS mutations. Crit Rev Oncol Hematol 85: 45-81, 2013.

5. Lim KH, Song MH and Baek KH: Decision for cell fate: Deubiquitinating enzymes in cell cycle checkpoint. Cell Mol Life Sci 73: 1439-1455, 2016.

6. Zhou MJ, Chen FZ and Chen HC: Ubiquitination involved enzymes and cancer. Med Oncol 31: 93, 2014.

7. Rickman KA, Lach FP, Abhyankar A, Donovan FX, Sanborn EM, Kennedy JA, Sougnez C, Gabriel SB, Elemento O, Chandrasekharappa SC, et al: Deficiency of UBE2T, the E2 ubiquitin ligase necessary for FANCD2 and FANCI ubiquitination, causes FA-T subtype of fanconi anemia. Cell Rep 12: 35-41, 2015.

8. Alpi A, Langevin F, Mosedale G, Machida YJ, Dutta A and Patel KJ: UBE2T, the Fanconi anemia core complex, and FANCD2 are recruited independently to chromatin: A basis for the regulation of FANCD2 monoubiquitination. Mol Cell Biol 27: 8421-8430, 2007.

9. Joenje $\mathrm{H}$ and Patel KJ: The emerging genetic and molecular basis of Fanconi anaemia. Nat Rev Genet 2: 446-457, 2001.

10. Weiser MR: AJCC 8th edition: Colorectal cancer. Ann Surg Oncol 25: 1454-1455, 2018

11. Livak KJ and Schmittgen TD: Analysis of relative gene expression data using real-time quantitative PCR and the 2(-Delta Delta C(T)) method. Methods 25: 402-408, 2001.

12. Lee YT, Tan YJ and Oon CE: Molecular targeted therapy: Treating cancer with specificity. Eur J Pharmacol 834: 188-196, 2018.

13. Nikolaou S, Qiu S, Fiorentino F, Rasheed S, Tekkis $\mathrm{P}$ and Kontovounisios C: Systematic review of blood diagnostic markers in colorectal cancer. Tech Coloproctol 22: 481-498, 2018.

14. Bhatti I, Patel M, Dennison AR, Thomas MW and Garcea G: Utility of postoperative CEA for surveillance of recurrence after resection of primary colorectal cancer. Int J Surg 16: 123-128, 2015.

15. Tsilimigras DI, Ntanasis-Stathopoulos I, Bagante F, Moris D, Cloyd J, Spartalis E and Pawlik TM: Clinical significance and prognostic relevance of KRAS, BRAF, PI3K and TP53 genetic mutation analysis for resectable and unresectable colorectal liver metastases: A systematic review of the current evidence. Surg Oncol 27: 280-288, 2018.
16. Raskov H, Pommergaard HC, Burcharth $\mathbf{J}$ and Rosenberg J: Colorectal carcinogenesis-update and perspectives. World J Gastroenterol 20: 18151-18164, 2014.

17. Bassermann F, Eichner R and Pagano M: The ubiquitin proteasome system-implications for cell cycle control and the targeted treatment of cancer. Biochim Biophys Acta 1843: 150-162, 2014.

18. Teixeira LK and Reed SI: Ubiquitin ligases and cell cycle control. Annu Rev Biochem 82: 387-414, 2013.

19. Alpi AF, Chaugule V and Walden $\mathrm{H}$ : Mechanism and disease association of E2-conjugating enzymes: Lessons from UBE2T and UBE2L3. Biochem J 473: 3401-3419, 2016.

20. Hira A, Yoshida K, Sato K, Okuno Y, Shiraishi Y, Chiba K, Tanaka H, Miyano S, Shimamoto A, Tahara H, et al: Mutations in the gene encoding the E2 conjugating enzyme UBE2T cause Fanconi anemia. Am J Hum Genet 96: 1001-1007, 2015.

21. Longerich S, San Filippo J, Liu D and Sung P: FANCI binds branched DNA and is monoubiquitinated by UBE2T-FANCL. J Biol Chem 284: 23182-23186, 2009.

22. Kelsall IR, Langenick J, MacKay C, Patel KJ and Alpi AF: The Fanconi anaemia components UBE2T and FANCM are functionally linked to nucleotide excision repair. PLoS One 7: e36970, 2012.

23. Ueki T, Park JH, Nishidate T, Kijima K, Hirata K, Nakamura Y and Katagiri T: Ubiquitination and downregulation of BRCA1 by ubiquitin-conjugating enzyme E2T overexpression in human breast cancer cells. Cancer Res 69: 8752-8760, 2009.

24. Gong YQ, Peng D, Ning XH, Yang XY, Li XS, Zhou LQ and Guo YL: UBE2T silencing suppresses proliferation and induces cell cycle arrest and apoptosis in bladder cancer cells. Oncol Lett 12: 4485-4492, 2016.

25. Hao J, Xu A, Xie X, Hao J, Tian T, Gao S, Xiao X and He D: Elevated expression of UBE2T in lung cancer tumors and cell lines. Tumour Biol 29: 195-203, 2008.

26. Yu H, Xiang P, Pan Q, Huang Y, Xie N and Zhu W: Ubiquitin-conjugating enzyme E2T is an independent prognostic factor and promotes gastric cancer progression. Tumour Biol 37: 11723-11732, 2016.

27. Hu W, Xiao L, Cao C, Hua S and Wu D: UBE2T promotes nasopharyngeal carcinoma cell proliferation, invasion, and metastasis by activating the AKT/GSK3 $\beta / \beta$-catenin pathway. Oncotarget 7 : 15161-15172, 2016

28. Wu M, Li X, Huang W, Chen Y, Wang B and Liu X: Ubiquitin-conjugating enzyme E2T(UBE2T) promotes colorectal cancer progression by facilitating ubiquitination and degradation of p53. Clin Res Hepatol Gastroenterol, Jul 29, 2020 (Online ahead of print).

29. Evan GI and Vousden KH: Proliferation, cell cycle and apoptosis in cancer. Nature 411: 342-348, 2001.

30. Kastan MB and Bartek J: Cell-cycle checkpoints and cancer. Nature 432: 316-323, 2004.

31. Sica V, Bravo-San Pedro JM, Stoll G and Kroemer G: Oxidative phosphorylation as a potential therapeutic target for cancer therapy. Int J Cancer 146: 10-17, 2020

32. Slattery ML, Mullany LE, Wolff RK, Sakoda LC, Samowitz WS and Herrick JS: The p53-signaling pathway and colorectal cancer: Interactions between downstream p53 target genes and miRNAs. Genomics 111: 762-771, 2019.

33. Bashir T and Pagano M: Aberrant ubiquitin-mediated proteolysis of cell cycle regulatory proteins and oncogenesis. Adv Cancer Res 88: 101-144, 2003.

34. Patra KC and Hay N: The pentose phosphate pathway and cancer. Trends Biochem Sci 39: 347-354, 2014.

35. Mamrak NE, Shimamura A and Howlett NG: Recent discoveries in the molecular pathogenesis of the inherited bone marrow failure syndrome Fanconi anemia. Blood Rev 31: 93-99, 2017.

This work is licensed under a Creative Commons Attribution-NonCommercial-NoDerivatives 4.0 International (CC BY-NC-ND 4.0) License. 